University of Wollongong

Research Online

Australian Institute for Innovative Materials -

Papers

Australian Institute for Innovative Materials

$1-1-2014$

Magnetism and magnetocaloric effect of Mn0.98Fe0.02CoGe

Q Y. Ren

University of New South Wales

W D. Hutchison

University of New South Wales

Jianli Wang

University of Wollongong, jianli@uow.edu.au

S Munoz Perez

University of New South Wales

J M. Cadogan

University of New South Wales, j.cadogan@unsw.edu.au

See next page for additional authors

Follow this and additional works at: https://ro.uow.edu.au/aiimpapers

Part of the Engineering Commons, and the Physical Sciences and Mathematics Commons

Research Online is the open access institutional repository for the University of Wollongong. For further information contact the UOW Library: research-pubs@uow.edu.au 


\title{
Magnetism and magnetocaloric effect of Mn0.98Fe0.02CoGe
}

\author{
Abstract \\ The crystallographic and magnetic properties of Mn0.98Fe0.02CoGe have been investigated by $\mathrm{X}$-ray \\ diffraction, dc magnetization and neutron diffraction over the temperature range $20-450 \mathrm{~K}$. The \\ temperature dependence of the phase fractions of the orthorhombic and hexagonal phases is described \\ well by a Gaussian distribution. The Mn0.98Fe0.02CoGe sample exhibits a first-order magneto-structural \\ transition centred at TMS $\sim 297 \mathrm{~K}$ of FWHM $\sim 37 \mathrm{~K}$ with a magnetic entropy change of $-\Delta S M=24(1) \mathrm{J}$ \\ $\mathrm{kg}-1 \mathrm{~K}-1$ for $\Delta \mathrm{B}=0-5 \mathrm{~T}$. Neutron diffraction indicates a ferromagnetic orthorhombic structure below TMS \\ with only the Mn carrying magnetic moment (3.98(6) $\mu \mathrm{B})$ at $20 \mathrm{~K}$. The sample is paramagnetic in the \\ hexagonal phase above TMS. \\ Keywords \\ $98 \mathrm{fe} 0,02 \mathrm{coge}$, magnetocaloric, magnetism, mn0, effect \\ Disciplines \\ Engineering | Physical Sciences and Mathematics \\ Publication Details \\ Ren, Q. Y., Hutchison, W. D., Wang, J. L., Perez, S. Munoz., Cadogan, J. M. \& Campbell, S. J. (2014). \\ Magnetism and magnetocaloric effect of Mn0.98Fe0.02CoGe. Physica Status Solidi A: Applications and \\ Materials Science, 211 (5), 1101-1105.

\section{Authors} \\ Q Y. Ren, W D. Hutchison, Jianli Wang, S Munoz Perez, J M. Cadogan, and S J. Campbell
}




\title{
Magnetism and the magnetocaloric effect of $\mathrm{Mn}_{0.98} \mathrm{Fe}_{0.02} \mathrm{CoGe}$
}

\author{
Q. Y. Ren ${ }^{*, 1}$, W. D. Hutchison ${ }^{1}$, J. L. Wang ${ }^{2,3}$, S. Munoz Pérez ${ }^{1}$, J. M. Cadogan ${ }^{1}$ and S. J. Campbell ${ }^{1}$ \\ ${ }^{1}$ School of Physical, Environmental and Mathematical Sciences, The University of New South Wales, Canberra, ACT 2600, Australia \\ ${ }^{2}$ Institute for Superconductivity and Electronic Materials, University of Wollongong, Wollongong, NSW 2522, Australia \\ ${ }^{3}$ Bragg Institute, Australian Nuclear Science and Technology Organisation, Lucas Heights, NSW 2234, Australia
}

Received ZZZ, revised ZZZ, accepted ZZZ

Published online ZZZ (Dates will be provided by the publisher.)

Keywords Magnetocaloric effect, magneto-structural transition, temperature-dependent x-ray diffraction, neutron diffraction

* Corresponding author: Qingyong.Ren@student.adfa.edu.au

The crystallographic and magnetic properties of $\mathrm{Mn}_{0.98} \mathrm{Fe}_{0.02} \mathrm{CoGe}$ have been investigated by $\mathrm{x}$-ray diffraction, dc magnetisation and neutron diffraction over the temperature range $20-450 \mathrm{~K}$. The temperature dependence of the phase fractions of the orthorhombic and hexagonal phases are described well by a Gaussian distribution of FWHM 36(2) K. The $\mathrm{Mn}_{0.98} \mathrm{Fe}_{0.02} \mathrm{CoGe}$ sam- ple exhibits a first-order magneto-structural transition at $T_{\mathrm{MS}} \sim 296.9(6) \mathrm{K}$ with a magnetic entropy change of $\Delta S_{\mathrm{M}}=24.24 \mathrm{~J} / \mathrm{kg} \cdot \mathrm{K}$ for $\Delta B=0-5 \mathrm{~T}$. Neutron diffraction indicates a ferromagnetic orthorhombic structure below $T_{\mathrm{MS}}$ with only the $\mathrm{Mn}$ carrying magnetic moment (3.98(6) $\mu_{\mathrm{B}} /$ f.u.) at $20 \mathrm{~K}$. The sample is paramagnetic in the hexagonal phase above $T_{\mathrm{MS}}$.
1 Introduction Magnetic refrigeration techniques based on the magnetocaloric effect (MCE) are attracting increasing attention as an alternative to conventional vapour-cycle refrigeration, for energy-saving and environmental reasons [1]. For widespread commercial applications, materials that exhibit giant magnetocaloric effect are required. Most giant MCE materials exhibit a first-order magnetic phase transition with coinciding crystallographic and magnetic transitions (magneto-structural transition) for which a magnetic field can induce changes of the magnetic and lattice entropies simultaneously [1-4]. As with the $\mathrm{R}_{5}(\mathrm{Si}, \mathrm{Ge})_{4}$ system, MnCoGe-based alloys exhibit a magneto-structural transition which could offer the potential for magnetocaloric refrigeration $[5,6]$.

MnCoGe-based alloys can form two stable phases: the nominally low temperature orthorhombic TiNiSi-type structure, with space group Pnma, and the nominally high temperature hexagonal $\mathrm{Ni}_{2}$ In-type structure, with space group $\mathrm{P6}_{3} / \mathrm{mmc}$ [7]. A crystallographic structural (martensitic) transition from the hexagonal phase to orthorhombic phase in pure MnCoGe occurs around $T_{\text {str }} \sim 650 \mathrm{~K}$ [8]. For the parent MnCoGe compound, the ferromagnetic ordering temperature is $T_{\mathrm{C}}^{\text {orth }} \sim 345 \mathrm{~K}$. Partial substitution of $\mathrm{Mn}$ for Co stablises the hexagonal phase at low temperature, and the ferromagnetic ordering temperature was obtained to be
$T_{\mathrm{C}}^{\text {hex }} \sim 275 \mathrm{~K}$ [9]. Clearly the structural transition temperature $T_{\text {str }}$ is much higher than the Curie temperature of either phase and, therefore no magneto-structural transition can be obtained in the pure compound. A key factor, however, in the suitability of MnCoGe-based alloys is that the structural transition temperature can be tuned into the temperature range of $T_{\mathrm{C}}^{\text {hex }}-T_{\mathrm{C}}^{\text {orth }}$, yielding a magneto-structural transition and thereby a giant magnetocaloric effect [10, 11]. Several methods have been applied to drive $T_{\text {str }}$ into this temperature window including the application of external pressure [12], the substitution of different elements [13], the introduction of vacancies [10] and/or interstitial atoms [5].

$\mathrm{Fe}$ is an often used substitution to tune martensitic transition temperatures $[4,14,15]$. In the present case Fe is also a suitable substitute for $\mathrm{Mn}$ since differences in the atomic radii $\left(\mathrm{r}_{\mathrm{Mn}}=1.35 \AA ; \mathrm{r}_{\mathrm{Fe}}=1.26 \AA\right)$ lead to reduced lattice parameters, which in turn tend to stabilise the hexagonal phase at lower temperatures, thereby decreasing $T_{\text {str }}$ to within the required temperature range. Three investigations of MnCoGe doped with Fe have been reported recently [16-18]. In order to fully understand the effects of Fe substitution for $\mathrm{Mn}$ on the magnetic and structural properties, we have carried out a detailed investigation of the structural and magnetic properties of $\mathrm{Mn}_{0.98} \mathrm{Fe}_{0.02} \mathrm{CoGe}$ 
by dc magnetisation, complemented with temperature dependent $\mathrm{x}$-ray and neutron diffraction measurements.

2 Experimental details The polycrystalline $\mathrm{Mn}_{0.98}$ $\mathrm{Fe}_{0.02} \mathrm{CoGe}$ sample was prepared by repeated argon-arc melting of stoichiometric amounts of Fe, Co, Ge plus Mn (reduced using dilute acid solution). The mass loss of Mn during melting was compensated by adding 3\% excess of $\mathrm{Mn}$ to the starting material. The sample was characterised by $\mathrm{x}$-ray powder diffraction (XRD) measurements in the temperature range between $20 \mathrm{~K}$ and $300 \mathrm{~K}$ using a PANalytical diffractometer with $\mathrm{Cu}-\mathrm{K}_{\alpha}$ radiation. The magnetisation was measured in a magnetic field of $0.01 \mathrm{~T}$ over the temperature range from $5 \mathrm{~K}$ to $350 \mathrm{~K}$ using a Physical Properties Measurement System (PPMS) from Quantum Design. In addition, the field dependence of the isothermal magnetisation was measured in magnetic fields up to $8 \mathrm{~T}$, from $205 \mathrm{~K}$ to $320 \mathrm{~K}$ in steps of $5 \mathrm{~K}$. Neutron diffraction experiments were carried out over the temperature range 5 - $450 \mathrm{~K}$ using the High-Intensity Powder Diffractometer Wombat $(\lambda=2.4205 \AA)$ at the OPAL reactor, Australia. All Rietveld refinements were carried out using the FullProf package [19, 20].

\section{Results and Discussion}

3.1 X-ray Diffraction Selected XRD patterns for $\mathrm{Mn}_{0.98} \mathrm{Fe}_{0.02} \mathrm{CoGe}$ obtained at different temperatures are shown in Fig. 1a. Coexistence of the hexagonal and orthorhombic phases is found above $255 \mathrm{~K}$ and up to $300 \mathrm{~K}$. In addition, a clear tendency for an increase in the fraction of the hexagonal phase is observed within this temperature range together with a commensurate decrease in the orthorhombic phase. The temperature dependence of the phase fractions of the hexagonal and orthorhombic phases was obtained from the Rietveld refinements (see Fig. 1b). It is worth noting that a small amount of hexagonal phase remains below the structural transition range, e.g. 2.4(3) wt\% hexagonal phase at $20 \mathrm{~K}$, as is also seen in $\mathrm{MnCoGeB}_{0.02}$ [5]. The presence of the hexagonal phase below $T_{\text {str }}$ was considered as a favourable factor for the structural transition [5]. In other words, this small hexagonal phase residual provides nuclei for the transformation from the orthorhombic phase to the hexagonal phase.

The inset of Fig. 1b shows the evolution of the orthorhombic and hexagonal phases as a function of temperature between $250 \mathrm{~K}$ and $300 \mathrm{~K}$. It was considered that the phase transition distribution follows the Gaussian function [21]:

$$
v(T, H)=\frac{1}{\sigma \sqrt{2 \pi}} \cdot e^{-\left(\frac{T-T_{s t r}-m H}{\sqrt{2} \sigma}\right)^{2}}
$$

where $m=d T_{\text {str }} / d H, T_{\text {str }}$ is the peak temperature in the Gaussian distribution (considered as the transition temperature) and $\sigma$ is the standard deviation. The full width at half maximum (FWHM) is calculated from $\sigma$ as follows:

$\mathrm{FWHM}=2 \sqrt{2 \ln 2} \cdot \sigma$
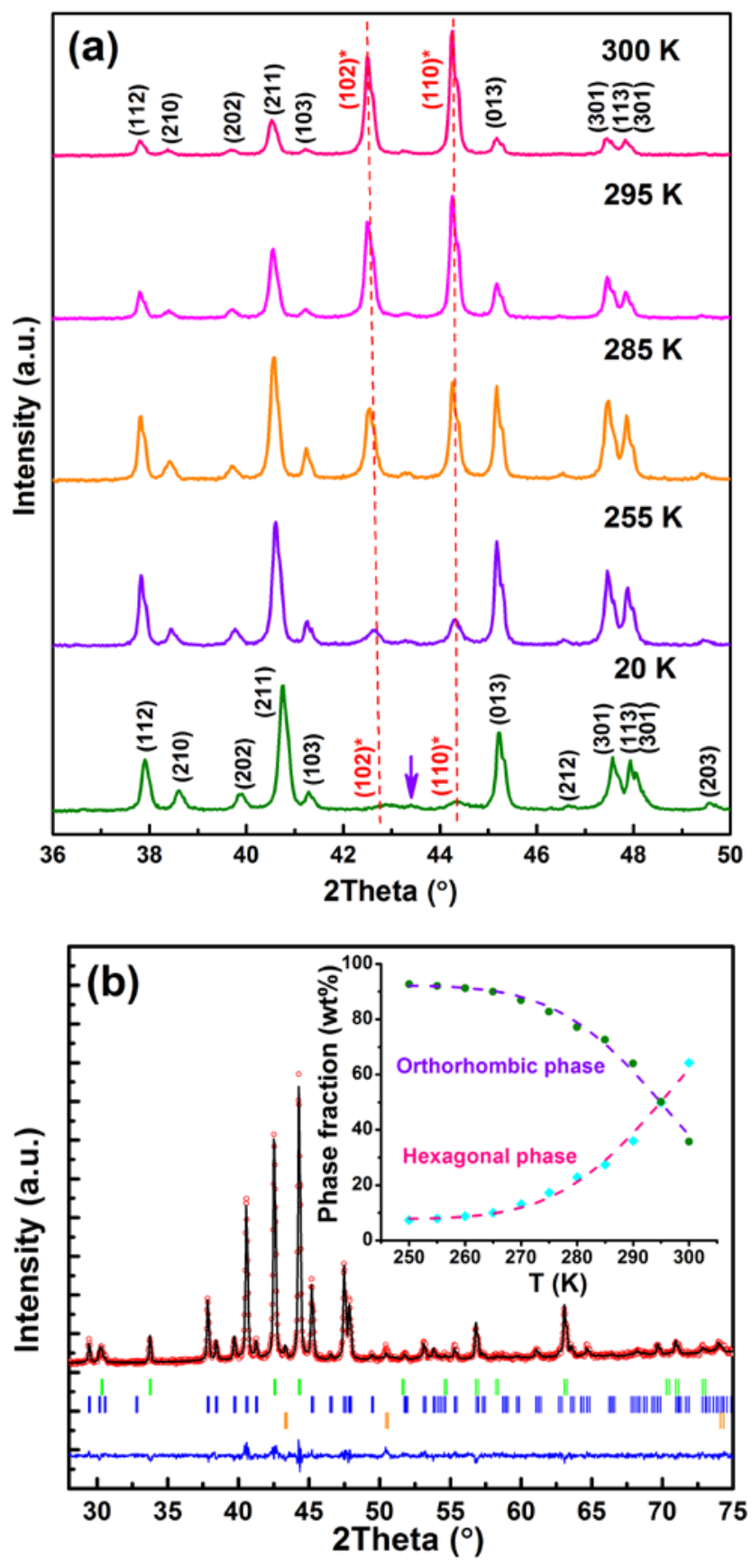

Figure 1 (a) Selected XRD patterns measured in zero-field upon heating at $20 \mathrm{~K}, 255 \mathrm{~K}, 285 \mathrm{~K}, 295 \mathrm{~K}$ and $300 \mathrm{~K}$ for $\mathrm{Mn}_{0.98} \mathrm{Fe}_{0.02} \mathrm{CoGe}$. The $h k l$ Miller indices of the hexagonal and orthorhombic phases are designated with and without an asterisk $(*)$, respectively. The small peak indicated by the arrow is from copper in the sample holder. (b) The Rietveld refinement of the XRD pattern obtained at $295 \mathrm{~K}$. The horizontal rows of Bragg markers (top to bottom) represent: the hexagonal (green), orthorhombic (blue) and copper (orange) phases. The inset shows the temperature dependence of the phase fractions (wt\%) of the hexagonal and orthorhombic structures between $250 \mathrm{~K}$ and $300 \mathrm{~K}$. The dashed lines represent fits to the data using Eq. (4) as discussed in the text. 
The weight phase fractions as a function of temperature can be obtained by integrating Eq. (1):

$$
f(T, H)=\int_{-\infty}^{T} v\left(T^{\prime}, H\right) d T^{\prime}
$$

Considering the residual fraction of the hexagonal phase at low temperatures as mentioned above, and given the partial inhomogeneity, Eq. (1) can be rewritten as:

$$
f(T, H)=(100-C) \cdot \int_{-\infty}^{T} v\left(T^{\prime}, H\right) d T^{\prime}+C
$$

where $\mathrm{C}$ represents a constant phase fraction. The fits to the data using Eq. (4) are shown in the inset of Fig. 1b (dashed lines). The transition temperature was determined to be $T_{\text {str }} \sim 296.9(6) \mathrm{K}$. This decrease in the structural transformation temperature occurs as a consequence of the smaller Mn-Mn distance due to the "chemical pressure" resulting from the substitution of transition metals which is helpful in stabilising the hexagonal $\mathrm{Ni}_{2}$ In-type structure, similar to the effect of the substitution of $\mathrm{V}$ for $\mathrm{Mn}$ in MnCoGe [11]. The FWHM of the phase transformation was obtained to be $36(2) \mathrm{K}$.

3.2 Magnetisation and Neutron Diffraction The temperature dependence $(5-350 \mathrm{~K})$ of the magnetisation of $\mathrm{Mn}_{0.98} \mathrm{Fe}_{0.02} \mathrm{CoGe}$ is shown in Fig. 2. The measurements were performed in a magnetic field of $0.01 \mathrm{~T}$, after cooling in zero applied field, firstly by stepping up in temperature (ZFC), followed by stepping down (FC). An abrupt magnetic state change from a low temperature ferromagnetic state to a high temperature paramagnetic phase with a large drop in magnetisation is observed.

The magnetic-state-change temperatures were determined as $300 \mathrm{~K}$ upon heating and $290 \mathrm{~K}$ upon cooling, through differentiation of the magnetisation curves versus temperature (i.e. $\mathrm{d} M / \mathrm{d} T$ versus $T$ ) as shown in the inset of Fig. 2. Both values lie in the temperature window, and locate at the right and left sides of $T_{\text {str }}$ respectively. Therefore a phase transformation between paramagnetic hexagonal phase and ferromagnetic orthorhombic phase occurs, resulting in a switch in different magnetic states. In other words, this phase transformation is a structural transition with a magnetic state change, that is, the magneto-structural transition at occurring at $T_{\mathrm{MS}} \sim 296.9(6)$ $K$. The presence of thermal hysteresis between the cooling and heating cycles in Fig. 2 confirms the first-order nature of this magneto-structural (martensitic) transition which corresponds to the austenitic transition $T_{\mathrm{A}} \sim 300 \mathrm{~K}$ and the martensitic transitions $T_{\mathrm{M}} \sim 290 \mathrm{~K}$. More importantly, a large MCE is expected around $T_{\mathrm{MS}}$.

In Fig. 3 we show the neutron diffraction (ND) patterns collected at $20 \mathrm{~K}$ and $365 \mathrm{~K}$; these temperatures were selected in order to study the crystallographic and magnetic structures of $\mathrm{Mn}_{0.98} \mathrm{Fe}_{0.02} \mathrm{CoGe}$ below and above the magneto-structural transition. The magnetic structure was determined using irreducible representational analysis [22, 23]. At $20 \mathrm{~K}$, the main crystallographic phase is the orthorhombic phase, in agreement with our XRD studies.

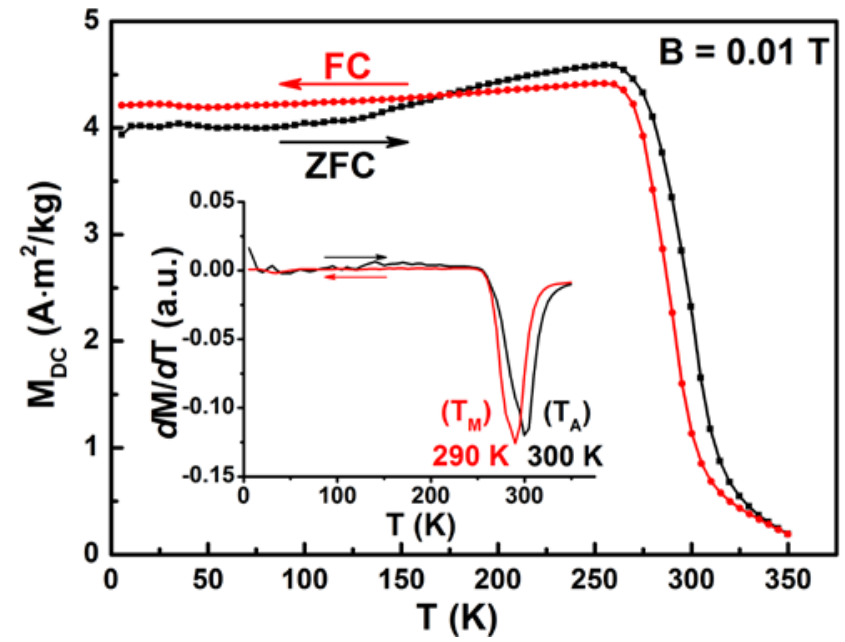

Figure 2 Magnetisation curves of $\mathrm{Mn}_{0.98} \mathrm{Fe}_{0.02} \mathrm{CoGe}$ as a function of temperature obtained with an applied magnetic field of $0.01 \mathrm{~T}$ on heating and cooling. The inset shows the derivative curves which were used to determine the phase transition temperatures.

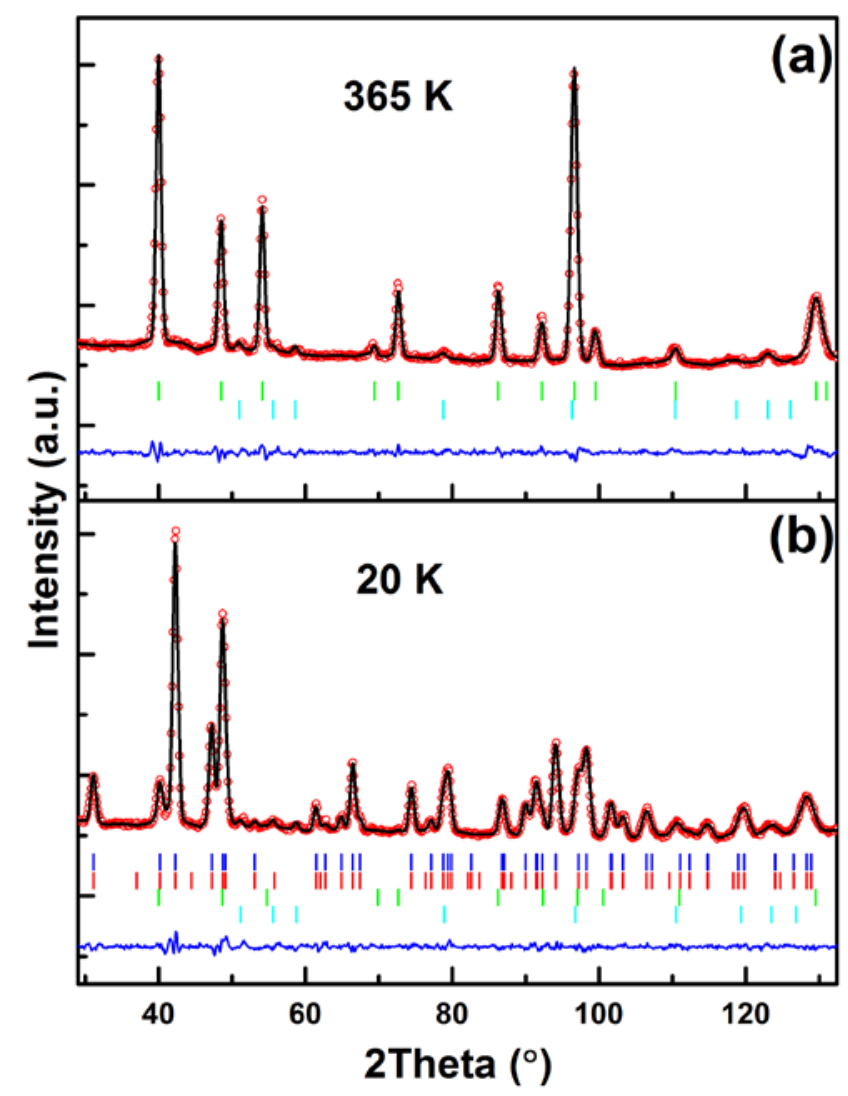

Figure 3 Rietveld refinements of the neutron diffraction patterns for $\mathrm{Mn}_{0.98} \mathrm{Fe}_{0.02} \mathrm{CoGe}$ collected at (a) $365 \mathrm{~K}$ and (b) $20 \mathrm{~K}$. The horizontal rows of Bragg markers (top to bottom) represent: (blue) the nuclear structure of the orthorhombic phase; (red) the magnetic structure of the orthorhombic phase; (green) the nuclear structure of the hexagonal phase and (cyan) the impurity (CoO). 
The analysis of a suite of ND patterns collected below $T_{\mathrm{MS}}$ indicates that $\mathrm{Mn}_{0.98} \mathrm{Fe}_{0.02} \mathrm{CoGe}$ adopts a ferromagnetic structure below $T_{\mathrm{MS}}$ (e.g. $20 \mathrm{~K}$ pattern of Fig. 3b). Furthermore these neutron refinements yield a magnetic structure consisting of ferromagnetic $\mathrm{Mn}$ aligned along the caxis, with a magnetic moment of 3.98(6) $\mu_{\mathrm{B}} /$ f.u. The Co mangetic moment is too small to be resolved. Indeed, refinement suggests it may be near zero. We note that while it is not unexpected that the estimated Mn moment should be somewhat smaller than the value of $4.13 \mu_{\mathrm{B}} / \mathrm{f}$.u. reported for the undoped MnCoGe compound [24] given the substitution of $\mathrm{Fe}$ is known to lower the average moment in these systems, the ND estimate for Mn is larger than the value derived from the saturated magnetisation for the sample at $5 \mathrm{~K}$ of $3.6(1) \mu_{\mathrm{B}} /$ f.u. Clearly there is an inconsistency here that will be the subject of further analysis, as is the apparently contradictory results of an NMR study [25]. However it is conceivable in the latter that the hyperfine field measured at the ${ }^{59}$ Co sites by NMR is predominantly a transferred field from the ferromagnetic Mn sublattice. At $365 \mathrm{~K}$, the main crystallographic phase is hexagonal and the compound is paramagnetic. A small amount of impurity (less than $4 \mathrm{wt} \%$, assigned as $\mathrm{CoO}$ ) was found in both neutron diffraction patterns but was not seen in the XRD patterns.

The field dependence of the isothermal magnetisation of $\mathrm{Mn}_{0.98} \mathrm{Fe}_{0.02} \mathrm{CoGe}$ was measured in both increasing and decreasing magnetic field $(\mathrm{B}=0-8 \mathrm{~T}$ ) within the temperature range of $205-320 \mathrm{~K}$ in steps of $5 \mathrm{~K}$. The magnetisation curves measured with increasing magnetic field are shown in Fig. 4. Below the magneto-structural transition temperature $T_{\mathrm{MS}} \sim 296.9(6) \mathrm{K}$, the magnetisation increases rapidly with increasing magnetic field, consistent with a ferromagnetic state while above $T_{\mathrm{MS}}$ the magnetisation change is less pronounced and the saturated magnetisation drops dramatically. All of these phenomena confirm a coupling between the structural and magnetic transitions in the $\mathrm{Mn}_{0.98} \mathrm{Fe}_{0.02} \mathrm{CoGe}$ sample.

3.3 Magnetocaloric Effect The isothermal magnetic entropy change $\Delta S_{\mathrm{M}}$ as a function of temperature for $\mathrm{Mn}_{0.98} \mathrm{Fe}_{0.02} \mathrm{CoGe}$ (see Fig. 5) was calculated based on the data of Fig. 4 using the Maxwell relation:

$$
\Delta S_{M}=\int_{0}^{B}\left(\frac{\partial M\left(T, B^{\prime}\right)}{\partial T}\right)_{B} d B^{\prime}
$$

The maximum isothermal magnetic entropies are obtained at $295.7 \mathrm{~K}$; this temperature corresponds to the transition temperature $T_{\mathrm{MS}} \sim 296.9(6) \mathrm{K}$. The maximum isothermal magnetic entropies at $295.7 \mathrm{~K}$ are: $-\Delta S_{\mathrm{M}}=10.09 \mathrm{~J} / \mathrm{kg} \cdot \mathrm{K}$ and $-\Delta S_{\mathrm{M}}=24.24 \mathrm{~J} / \mathrm{kg} \cdot \mathrm{K}$ for magnetic field changes of $\Delta B$ $=0-2 \mathrm{~T}$ and $0-5 \mathrm{~T}$, respectively. The value for the present $\mathrm{Mn}_{0.98} \mathrm{Fe}_{0.02} \mathrm{CoGe}$ sample for $\Delta B=0-5 \mathrm{~T}$ is larger than that reported for the $\mathrm{Mn}_{1-\mathrm{x}} \mathrm{Fe}_{\mathrm{x}} \mathrm{CoGe}$ series of compounds [16-18]. In addition, our $\mathrm{Mn}_{0.98} \mathrm{Fe}_{0.02} \mathrm{CoGe}$ sample is found to exhibit a MCE value comparable with the MCE values

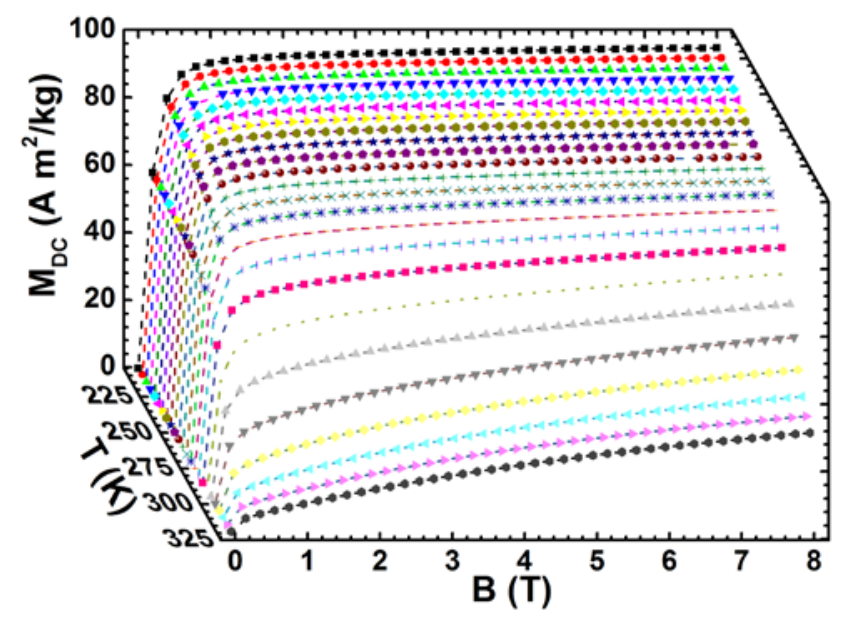

Figure 4 The magnetic field dependence of the isothermal magnetisation for $\mathrm{Mn}_{0.98} \mathrm{Fe}_{0.02} \mathrm{CoGe}(\mathrm{B}=0 \mathrm{~T}-8 \mathrm{~T})$ as measured in increasing magnetic field up to $8 \mathrm{~T}$ from $205 \mathrm{~K}$ to $320 \mathrm{~K}$ in $5 \mathrm{~K}$ intervals.

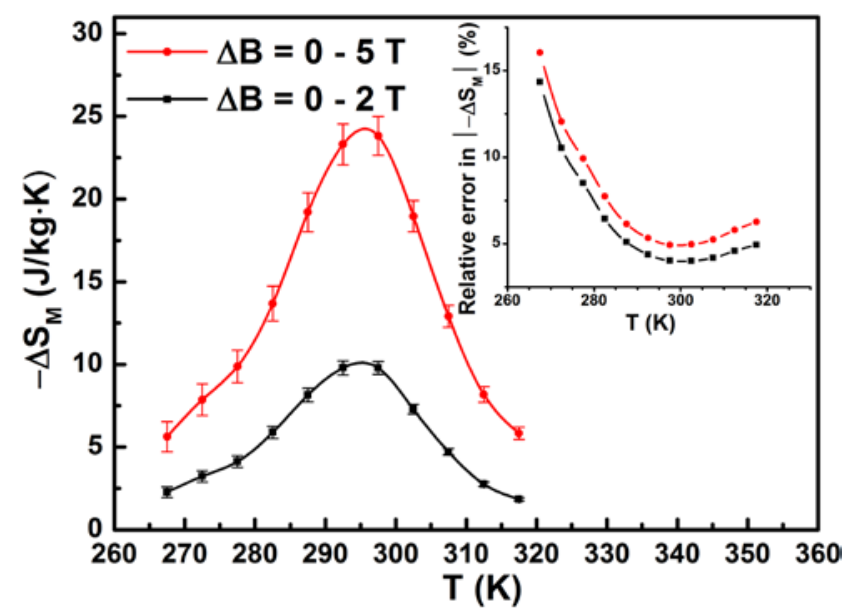

Figure 5 Temperature dependence of the isothermal magnetic entropy change $-\Delta S_{\mathrm{M}}$ calculated from the magnetisation isotherms obtained in an increasing magnetic field for $\mathrm{Mn}_{0.98} \mathrm{Fe}_{0.02} \mathrm{CoGe}$ with magnetic field changes of $0-2 \mathrm{~T}$ and $0-5 \mathrm{~T}$. Inset was the corresponding relative errors in $\left|-\Delta S_{\mathrm{M}}\right|(\%)$ calculated based on the Eq. (2.4b) in [27].

reported recently for $\mathrm{Tb}_{5} \mathrm{Si}_{2} \mathrm{Ge}_{2}(\sim 23.4 \mathrm{~J} / \mathrm{kg} \cdot \mathrm{K}$ at $118 \mathrm{~K}$ when a $10.2 \mathrm{kbar}$ pressure was applied) [26]. The relatively large MCE exhibited by our sample is due to the successful coupling of the magnetic and lattice structures as discussed above. As with other MnCoGe-based alloys, the magnetostructural transition of our sample also occurs around room temperature, thus offering potential for practical applications.

In addition, the assigned errors in Fig. 5 were calculated using Eq. (2.4b) in Ref. [27] (see the error bars shown 
in Fig. 5). Following [27], we assumed the accuracy of the magnetisation measurements at $0.5 \%$, the accuracy of the magnetic field at $0.1 \%$ and the errors for temperature as $0.1 \mathrm{~K}+0.001 \times T$. According to the inset in Fig. 5, the relative error in the $\left|-\Delta S_{\mathrm{M}}\right|$ is between $\sim 4 \%$ and $\sim 7 \%$ above $T_{\mathrm{MS}}$ and is significantly higher below $T_{\mathrm{MS}}$, similar to the trend shown by Gd [27]. However the values are smaller than that given in [27], because increased temperature step and magnetic field step lead to a decrease in the error.

4 Conclusions The structural and magnetic transitions of $\mathrm{Mn}_{0.98} \mathrm{Fe}_{0.02} \mathrm{CoGe}$ have been investigated by temperature dependent $\mathrm{x}$-ray diffraction, magnetisation and neutron diffraction measurements. Partial substitution of Fe for Mn in MnCoGe leads to a decrease in the hexagonal to orthorhombic structural transformation temperature compared with the parent MnCoGe compound, $T_{\text {str }} \sim 650$ $\mathrm{K}$ [8] and results in a magneto-structural transition of $T_{\mathrm{MS}}$ $\sim$ 296.9(5) $\mathrm{K}$ for $\mathrm{Mn}_{0.98} \mathrm{Fe}_{0.02} \mathrm{CoGe}$. . A large magnetic entropy change was obtained $\left(-\Delta S_{\mathrm{M}}=24.24 \mathrm{~J} / \mathrm{kg} \cdot \mathrm{K}\right.$ for $\Delta B=$ $0-5 \mathrm{~T})$. Analysis of the neutron diffraction patterns confirm a ferromagnetic orthorhombic phase at low temperature below $T_{\mathrm{MS}}$ with a paramagnetic hexagonal phase above $T_{\mathrm{MS}}$. Rietveld refinements indicate that the Mn sublattice has a magnetic moment of $\mu_{\mathrm{Mn}}=3.98(6) \mu_{\mathrm{B}} / \mathrm{f}$.u in the orthorhombic phase at $20 \mathrm{~K}$.

Acknowledgements This work was supported in part by grants from the Australian Research Council (DP110102386) and ARC LIEF grant (LE1001000177). QYR acknowledges a Research Training Scholarship from UNSW Canberra. The authors thank Dr A. J. Studer for assistance during the neutron diffraction experiments.

\section{References}

[1] E. Brück, J. Phys. D: Appl. Phys. 38, R381 (2005)

[2] V. K. Pecharsky and K. A. Gschneidner Jr, Phys. Rev. Lett. 78, 4494 (1997)

[3] T. Krenke, E. Duman, M. Acet, E. F. Wassermann, X. Moya, L. Manosa and A. Planes, Nat. Mater. 4, 450 (2005)

[4] E. Liu, W. Wang, L. Feng, W. Zhu, G. Li, J. Chen, H. Zhang, G. Wu, C. Jiang, $\mathrm{H}$. $\mathrm{Xu}$ and F. de Boer, Nature communications 3, 873 (2012)

[5] N. T. Trung, L. Zhang, L. Caron, K. H. J. Buschow and E. Brück, Appl. Phys. Lett. 96, 172504 (2010)

[6] T. Samanta, I. Dubenko, A. Quetz, S. Stadler and N. Ali, Appl. Phys. Lett. 101, 242405 (2012)
[7] V. Johnson, Inorg. Chem. 14, 1117 (1975)

[8] T. Kanomata, H. Ishigaki, T. Suzuki, H. Yoshida, S. Abe and T. Kaneko, J. Magn. Magn. Mater. 140-144, Part 1, 131 (1995)

[9] K. Koyama, M. Sakai, T. Kanomata and K. Watanabe, Jpn. J. Appl. Phys. 43, 8036 (2004)

[10] E. K. Liu, W. Zhu, L. Feng, J. L. Chen, W. H. Wang, G. H. Wu, H. Y. Liu, F. B. Meng, H. Z. Luo and Y. X. Li, Europhys. Lett. 91, 17003 (2010)

[11] S. C. Ma, Y. X. Zheng, H. C. Xuan, L. J. Shen, Q. Q. Cao, D. H. Wang, Z. C. Zhong and Y. W. Du, J. Magn. Magn. Mater. 324, 135 (2012)

[12] S. Nizioł, A. Ziẹba, R. Zach, M. Baj and L. Dmowski, J. Magn. Magn. Mater. 38, 205 (1983)

[13] J. B. A. Hamer, R. Daou, S. Özcan, N. D. Mathur, D. J. Fray and K. G. Sandeman, J. Magn. Magn. Mater. 321, 3535 (2009)

[14] V. K. Sharma, M. K. Chattopadhyay, S. K. Nath, K. J. S. Sokhey, R. Kumar, P. Tiwari and S. B. Roy, J. Phys.: Condens. Matter 22, 486007 (2010)

[15] Z. Wu, Z. Liu, H. Yang, Y. Liu, G. Wu and R. C. Woodward, Intermetallics 19, 445 (2011)

[16] G. J. Li, E. K. Liu, H. G. Zhang, Y. J. Zhang, J. L. Chen, W. H. Wang, H. W. Zhang, G. H. Wu and S. Y. Yu, J. Magn. Magn. Mater. 332, 146 (2013)

[17] T. Samanta, I. Dubenko, A. Quetz, S. Stadler and N. Ali, J. Magn. Magn. Mater. 330, 88 (2013)

[18] Z. Wang, Z. Nie, J. Zeng, R. Su, Y. Zhang, D. E. Brown, Y. Ren and Y. Wang, J. Alloys Compd. 577, 486 (2013)

[19] H. Rietveld, J. Appl. Crystallogr. 2, 65 (1969)

[20] J. Rodríguez-Carvajal, Physica B: Condensed Matter 192, 55 (1993)

[21] J. Liu, T. Gottschall, K. P. Skokov, J. D. Moore and O. Gutfleisch, Nat. Mater. 11, 620 (2012)

[22] E. Bertaut, Acta Cryst. A 24, 217 (1968)

[23] S. V. Gallego, E. S. Tasci, G. de la Flor, J. M. Perez-Mato and M. I. Aroyo, J. Appl. Crystallogr. 45, 1236 (2012)

[24] L. Song, O. Tegus, E. Brück, W. Dagula, T. J. Gortenmulder and K. H. J. Buschow, Magnetics, IEEE Transactions on 42, 3776 (2006)

[25] T. Kanomata, H. Ishigaki, K. Sato, M. Sato, T. Shinohara, F. Wagatsuma and T. Kaneko, J. Magn. Soc. Japan 23, 418 (1999)

[26] N. A. de Oliveira, J. Appl. Phys. 113, 033910 (2013)

[27] V. K. Pecharsky and K. A. Gschneidner Jr, J. Appl. Phys. 86, 565 (1999) 